\title{
Autowaves in a dc complex plasma confined behind a de Laval nozzle
}

\author{
M. A. Fink ${ }^{1}$, S. K. Zhdanov ${ }^{1}$, M. Schwabe ${ }^{1,2}$, M. H. Thoma ${ }^{1}$, H. Höfner ${ }^{1}$, H. M. Thomas ${ }^{1}$, And G. E. \\ MORFILL ${ }^{1}$ \\ ${ }^{1}$ Max-Planck-Institute for Extraterrestrial Physics, Giessenbachstr., 85748 Garching, Germany \\ ${ }^{2}$ Department of Chemical and Biomolecular Engineering, University of California, Berkeley, CA 94720, USA
}

PACS 52.27.Lw - Dusty or complex plasmas; plasma crystals

PACS 52.30.-q - Plasma dynamics and flow

\begin{abstract}
Experiments to explore stability conditions and topology of a dense microparticle cloud supported against gravity by a gas flow were carried out. By using a nozzle shaped glass insert within the glass tube of a dc discharge plasma chamber a weakly ionized gas flow through a de Laval nozzle was produced. The experiments were performed using neon gas at a pressure of $100 \mathrm{~Pa}$ and melamine-formaldehyde particles with a diameter of $3.43 \mu \mathrm{m}$. The capturing and stable global confining of the particles behind the nozzle in the plasma were demonstrated. The particles inside the cloud behaved as a single convection cell inhomogeneously structured along the nozzle axis in a tube-like manner. The pulsed acceleration localized in the very head of the cloud mediated by collective plasma-particle interactions and the resulting wave pattern were studied in detail.
\end{abstract}

Introduction. - Solid 'seeds', the dust- or microparticles incorporated into a weakly ionized gas, can fundamentally alternate the low frequency dynamics of the entire gas-plasma-particles system. During the last decades such kind of complex plasmas has been of great particular interest in complex system studies [1. In experiments with complex plasmas the microparticle component, acquiring large negative charges in the plasma, is strongly non-ideal. It is represented typically by particles of a $\mu \mathrm{m}$ to some tens of $\mu \mathrm{m}$ in size. The internal structure of complex plasmas is mostly determined by the mutual particle-particle interactions consisting of isotropic (repulsive) and anisotropic (wake-mediated) forces, as recent detailed studies demonstrated [2] 6 . The global configuration on a large scale is determined by the external confining forces, which are also an important structuring element of the complex plasma dynamics $7 \sqrt{9}$. The interplay of isotropic, anisotropic and external forces gives rise to different competing symmetries [10,12, phase transitions 13 17, wave patterns [18 26, and streaming instabilities [27,28, successfully observed and explored at the kinetic level. Interdisciplinary aspects of complex plasma studies are discussed in Ref. [29].

Usually, explaining the complex plasma dynamics, it is safe to assume that in complex plasmas the main struc- turing interplay is based on the charged particles-plasma electric forces feedback mechanism. The neutral gas in such dynamical scenarios is considered as a passive factor, resulting mainly in the frictional reduction of the kinetic energy of the particles. Many interesting results are known to be explained successfully based on such simplified models, in particular the dynamics of $2 \mathrm{~d}$ complex plasmas (see, e.g., 30] and the references therein). Many phenomena are also known in which all three components - gas, dust and plasma, - can play an equally important dynamical role as e.g. in a swirling mass of dust forming a funnel in tornados, or gustnados [31, or in swirling dust devils which are common in dry deserts [32. The dust devils on Earth, even small ones, can produce radio noise and electrical fields of the order of $10-100 \mathrm{kV} / \mathrm{m}$, enough to produce a breakdown in dry air [33].

In laboratory experiments with complex plasmas, the gas component can be successfully used to control and manipulate the particles enabling e.g. the rotation of the cloud as a whole under gas drag [4,34, allowing to mimic the magnetization of the heavy dust by making use of the complex plasma-neutral gas frictional coupling [35, or to study the particle cloud convection in response to gas creep [36, 37]. Using small particle clusters as a probe of the axial discharge field distribution is also another fa- 
vorable opportunity [6].

In this letter we report on the effects of levitating particles with a gas flow counteracting gravity. In some sense the situation in our plasma experiments resembles 'forced convection', in which transport of particles is induced by a gas stream in a number of well-known examples, e.g., fluidized beds [38, snow machines, or volcano clouds. Unlike conventional forced convection, in our experiments the microparticles cannot be stably levitated by the gas flow alone. The plasma influences significantly the particle dynamics. In particular, the particle cluster stably confined inside the de Laval nozzle behaves as a single convection 'supercell' (to some extent resembling a microparticle blob observed in [39]) with the plasma ions acting as a trigger of the marginal instability resulting in the observed autowave pattern 1

Experimental conditions. - Experiments were conducted using a laboratory version of the Plasmakristall-4 experimental device designed to produce complex plasmas inside the positive column of a high-voltage dc currentregulated discharge. During the experiment campaign the $35-\mathrm{cm}$ long and $3 \mathrm{~cm}$ diameter glass discharge tube with an incorporated transparent glass de Laval nozzle was mounted vertically. The gas inlet system and the vacuum control system were resided correspondingly in the bottom and in the top parts of the tube. In the experiment the input voltage was set at $U_{d c}=1500 \mathrm{~V}$. Neon plasma at a pressure of $100 \mathrm{~Pa}$ was sustained using a dc discharge at a current $I_{d c}=1.1 \mathrm{~mA}$. The electron density, electron temperature and the electric field strength of the discharge far away from the nozzle were estimated to be: $T_{e}=6 \mathrm{eV}, n_{e}=4 \cdot 10^{8} \mathrm{~cm}^{-3}, E=2.1 \mathrm{~V} / \mathrm{cm}(\mathrm{cf}$. [6, 41, 42]).

Using the microparticle supply system located in the vicinity of the cathode atop of the discharge tube and above the nozzle, monodisperse melamineformaldehyde particles with a diameter of $3.43 \mu \mathrm{m}( \pm 2 \%)$ and a mass of $m=3.2 \cdot 10^{-11} \mathrm{~g}$ were injected into the dc discharge plasma. Under those discharge conditions, as the charge estimates based on the modified Orbital Motion Limited (m-OML) model 43 and the Drift Motion Limited (DML) model 44 show, the expected microparticle charge might be of the order of $Z e=-4000|e|$ (here $e$ is the elementary charge). Using the adjustable gas flow controller, a series of experiments was performed at a fixed controllable value of the gas flow rate $(0.1-0.4 \mathrm{sccm})$ (see footnot 22), powerful enough to suspend microparticle clusters with a number density up to $\sim 10^{6} \mathrm{~cm}^{-3}$. The clusters

\footnotetext{
${ }^{1}$ According to M. Faraday's publication [40], the term auto-wave applies to the topology of the wave structure, its stability, as well as the nature of the wave patterns.

${ }^{2}$ Without any external modulation. Still, it is worth noting that adjusting the flow rate excites low-frequency oscillations along the axis of symmetry of the nozzle, see e.g. Ref. 45. The leading frequency $\simeq 2.5 \mathrm{~Hz}$ of the flux oscillations [6] happened to be much lower than the observed wave frequencies $\simeq 30 \mathrm{~Hz}$, see below. The absolute error of the flow controller was estimated to be $0.006 \mathrm{sccm}$.
}

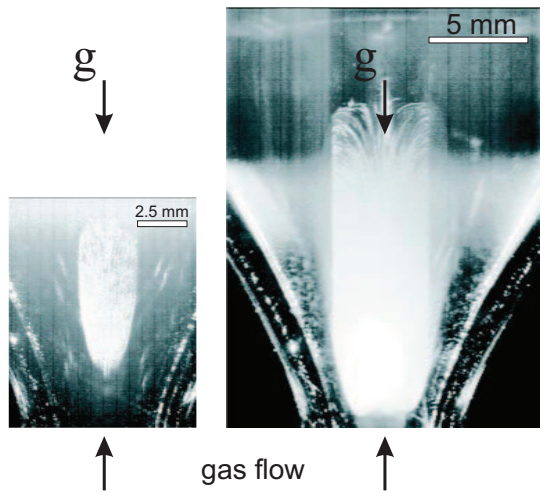

Fig. 1: A pellet-shaped circulation cell formed by the microparticles deep inside the nozzle. Fifty images were superimposed and equalized to emphasize the shape of the nozzle walls and the shape of the cloud. Circulation in the rarefied part of the tail of the cloud is seen as a 'fountain stream' of separated particle tracks. The incoming gas flow, counteracting gravity (g), is coming from the bottom: at $0.16 \mathrm{sccm}$, smaller cloud (left panel), and at $0.2 \mathrm{sccm}$, bigger cloud (right panel). The scales and the vertical positions were properly adjusted.

formed were illuminated from the bottom using a $100 \mu \mathrm{m}$ wide laser sheet. The particle dynamics was recorded by a CCD camera. The recording rate was set to 120 frames per second.

More technical details on the setup, the gas supply system, the laser illumination system and the image recording system can be found elsewhere [6].

A global design of the particle cluster. - In the course of the experiments the injected particles formed a long-living pellet-shaped cluster, see fig. 10 3 , with a major length of $5-15 \mathrm{~mm}$ depending on their total amount of particles. The nozzle walls, being overall transparent, are seen in this figure as a converging funnel-shaped set of bright laser light reflections. The illuminating laser light comes from below, partly scattered by entering the interior of the glass nozzle or reflected by the nozzle walls. A small cluster (as, e.g., shown to the left in fig. 1) with a transverse size less than the nozzle aperture is seen completely in transmitted light; the periphery parts of a bigger cluster are somewhat obscured (right panel in fig. 11). Still, some particle tracks on the cluster periphery are accessible to study 4 .

The microparticle cluster, being globally of a quite simple shape, has a complex internal structure, having quite the appearance of a comet. The latter is known to consist

\footnotetext{
${ }^{3}$ Equalization is a standard function in Mathcad 14.0. This function performs a histogram equalization and is used to enhance features of a picture by spreading the pixel intensities over a larger portion of the available spectrum.

${ }^{4}$ http://iopscience.iop.org/0295-5075/102/4/45001/media contains a movie with a spatial resolution of $33 \mu \mathrm{m} / \mathrm{px}$ (vertical) by $66 \mu \mathrm{m} / \mathrm{px}$ (horizontal). The real size of the field of view is $21.1 \times 15.8 \mathrm{~mm}^{2}$ (vertical $\times$ horizontal). The 240 frames recorded at $120 \mathrm{fps}$ are converted into a MPEG-4 movie at a frame rate of 15 fps.
} 


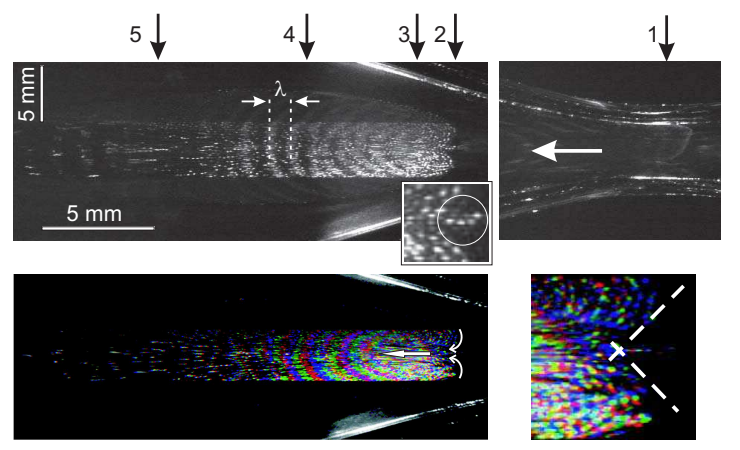

Fig. 2: (Color online) Top panels: the topology of the particle cluster and the (reconstructed) geometry of the nozzle. The main elements are: 1 . the nozzle throat; 2 . the little stable satellite cloud (highlighted by the circle in the inset); 3 . the dense compact nucleus of the main cluster; 4 . the wave pattern (spread apart along the bulk of the particle cluster); 5. the rarefied tail part (some particle strings are well visible). The space-scale is indicated by the white bar shown to the left. The gas flow through the nozzle $(0.4 \mathrm{sccm}$ for the given experiment), counteracting the force of gravity, is directed as a white arrow, drawn in the right image, indicates. The acceleration of gravity in the picture is pointing to the right, opposite the gas flow. The mean distance between the wave ridges was measured to be $\langle\lambda\rangle=(1.1 \pm 0.3) \mathrm{mm}$. Bottom, left: three consecutive RGB color-coded images are superimposed to emphasize the convective particle drift and the wave ridges propagating through. The white arrows indicate the dominant particle convection direction. Bottom, right: the enlarged image of the cluster nucleus with an emphasized cone-shaped indent (the dashed lines). The cone opening angle is $2 \theta \approx 130^{\circ}$.

of a nucleus, coma and tail, and our cloud (see fig. 2) also consists of a dense nucleus (unlike the comet, it is not solid, though), a bulk with a dancing dust making a rhythmic pattern, and a straight extended tail. All these structural elements are dynamically very delicately adjusted. Some of them, for instance, a cloud deformation caused by the gas head, a little satellite cloud, and the wave pattern, have a relatively simple explanation. The clusters were in an active, marginally unstable state 5 It was also noticeable that this complicated internal design took the same pattern for any of the explored gas flow rates.

An indentation and a satellite cloud. - In the apex of the cluster a crater was dug out by the gas head. It contains a little "satellite" cloud, i.e. a spatially separated compact particle cloud in close proximity to the main cluster. This cloud was always inside of the cone for the whole considered parameter range. The indentation cone and the satellite cloud are stable, at least structurally 6

The indentation cone is clearly seen in figs. 2, 3, The cone size is small (cone height/cone radius at the base $\approx$

\footnotetext{
${ }^{5}$ Such a state can lead to self-excitations in which natural modes can neither grow without bound nor decay to zero, see, e.g., [46].

${ }^{6}$ That is permanently restoring themselves approximately in the same shape.
}

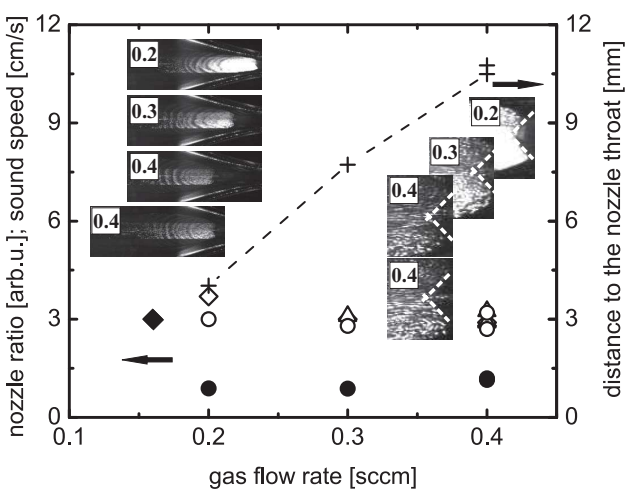

Fig. 3: The 'nozzle ratio' (solid dots) and the dust sound speed obtained with the same particle cluster for different gas flow rates. The speed of dust sound was measured by using the density fluctuations (open dots), the correlation methods (open triangles), and the traced particle tracks (open diamonds). The speed of sound obtained with a smaller cluster (fig.1 left panel) is shown for comparison (solid diamond). The stacked snapshots of the cluster $\left(\mathrm{FoV} \cong 1.6 \times 2.1 \mathrm{~cm}^{2}\right)$ are shown as insets to the left. To the right are shown the enlarged images of the cluster nuclei ordered in the same way. The imposed white dashed lines indicate the same cone opening angle as that in fig. 2 The numbers give the gas flow rates measured in standard $\mathrm{cu}-$ bic centimeter per minute. The (nearly) constant nozzle ratio $\eta=1.0 \pm 0.3$ indicates that the stronger gas flow pushes the entire cluster upwards away from the nozzle throat (the crosses and the dashed line).

$\frac{1}{2}$, the cone height is $\left.\simeq 0.5 \mathrm{~mm}\right)$. The satellite cloud is even smaller, and hence can serve as a convenient benchmark to study the cluster dynamics. The position $z_{s}$ of the center of mass of the satellite cloud can be obtained directly using the recorded cluster images. Then, suggesting that the axial forces are balanced, and making use of eq. (3) in Ref. 6, the 'effective' gravity was calculated by the force balance:

$$
m g_{\text {eff }}^{z}\left(z_{s}\right) \equiv m g+F_{E}^{z}\left(z_{s}\right)=m \gamma_{E p s} V_{g a s}^{z}\left(z_{s}\right)
$$

Here $m$ is the particle mass, $g$ is the free-fall acceleration on earth, $V_{\text {gas }}^{z}\left(z_{s}\right)$ is the axial gas flow velocity, $F_{E}^{z}\left(z_{s}\right)$ is the axial projection of the resultant electric force (effectively including e.g. the ion drag force) at the height $z=z_{s}$ above the nozzle throat, and $\gamma_{E p s}$ is the Epstein drag force coefficient 47, in our conditions $\gamma_{E p s}=227 \mathrm{~s}^{-1}$.

The result of the calculations is shown in fig. 3 as a 'nozzle ratio' introduced by the relationship

$$
\eta=\frac{g_{e f f}}{g} \frac{R^{2}}{j}
$$

where $R[\mathrm{~cm}]$ is the nozzle transverse radius taken on at the satellite position and $j[s c \mathrm{~cm}]$ is the gas flow rate.

The indentation surface is limited by the tracks of the particles gliding along it. Since the cluster above the surface is dense, the internal forces of the mutual interparticle interactions cannot be neglected. Let us consider a simple 


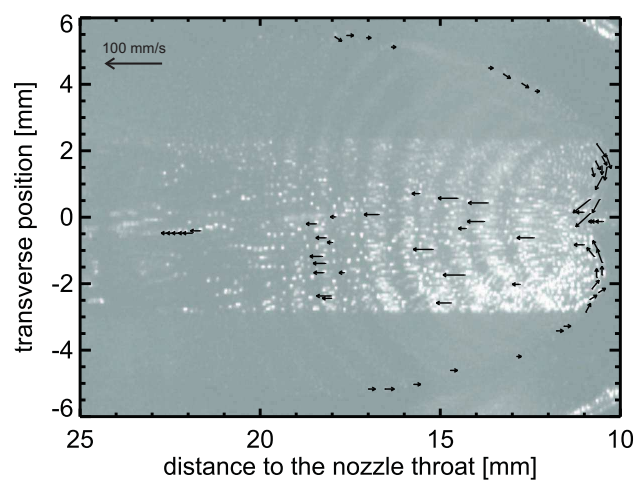

Fig. 4: A single-frame velocity vector field plot of the cycling particles at $0.4 \mathrm{sccm}$. A few selected particle positions are shown to emphasize the main drift directions: a relatively weak rightward periphery drift is replaced with a rather fast particle rotation located inside of the cluster head, which, in turn, is replaced by the central-part leftward drift, completing the circulation loop. The image of the particle cluster is shown in the background.

hydrodynamic model of a 'liquid with pressure', introducing a pressure force (per particle) by $\mathbf{F}_{p}=-n^{-1} \nabla p$, where $n$ is the cluster density, and $p$ is the pressure. At the surface the longitudinal component of the pressure force has to be completely balanced but the longitudinal velocity component is not zero, and, vise versa, transversely to the surface the pressure force is not zero but the transverse velocity has to vanish by definition. Giving the small size of the indentation, it is reasonable to assume that eq. (1) is valid also along the surface at the position of the satellite. Then it follows approximately that 7

$$
F_{p}^{\perp} \simeq m \gamma_{E p s} V_{d}^{\|} \cot \theta,
$$

where $V_{d}^{\|}$is the particle drift velocity and $\theta$ is the indentation cone angle. Relationship (3) has a rather simple physical meaning: In the framework of our model loss of mechanical energy due to friction is to be restored by work of pressure. Note also that in our conditions when

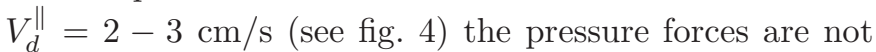
small, about $F_{p}^{\perp} / m g=(20-30) \%$. The forces necessary to accelerate the particles in the waves are basically of the same order.

A wave pattern and an instability driving mechanism. - The structure, accelerating the particles, is located in the cluster nucleus only $(0.5-1) \mathrm{mm}$ apart from the center of the satellite cloud where the particles are nearly immobile. It accelerates the microparticles up to the velocity $(2-3) \mathrm{cm} / \mathrm{s}$ practically from rest. Note that in our conditions the electron screening length $\lambda_{D e} \simeq 1 \mathrm{~mm}$ is of the order of the electron mean free path $\lambda_{e} \simeq 1 \mathrm{~mm}$

\footnotetext{
${ }^{7}$ The transverse $(\perp$-labeled $)$ and the longitudinal (\|-labeled) components of any vector-field $\mathbf{A}$ are introduced through its radial $r$ and axial $z$ projections by the relationships: $A^{\perp}=A^{z} \sin \theta+A^{r} \cos \theta$, $A^{\|}=A^{z} \cos \theta-A^{r} \sin \theta$
}

(Ne at $100 \mathrm{~Pa}$ ), that is of the size of the accelerating structure. Despite the expected increase of the plasma density deep inside the nozzle, a significant change in the screening length is not expected because the mean kinetic temperature of the electrons increases in the discharge constriction as well [48,49. The electrons are not in equilibrium with the electric field 48 .

The stable entrainment of particles in the upward gas flow is replaced then by a strong oscillatory motion. Qualitatively, the main stages, repeating in cycle, are as follows: a local sharp pulsed particle acceleration, then a drift directed upwards, momentary stop, short-time backward motion, etc. followed by the formation and downstream (to gas flow) propagation of the wave ridges well recognizable even in the raw cluster images (see fig. 21). To help recognizing the wave dynamics further, the cluster images were color-coded in the following manner. Three consecutive images were taken from the sequence, and then to every of them a RGB scale was attributed 8 Afterwards they were combined in one RGB-image shown in the bottom panel of fig. 2 Note that the white color emphasizes the equally bright 'immobile' pixels, e.g. those at the image edges where the 'immobile' light reflections are located. Whereas the 'mobile' pixels have taken the color red, green, or blue, performing a colored map.

The propagation of the strong regular wave ridges is accompanied by the excitation of noise easily identified as cluster density fluctuations. The results of the different noise spectra measurements (e.g. the analysis of the density fluctuation 8, 25] or the direct particle tracking methods [50]) agree reasonably well with each other, and with propagation analysis of the wave ridges [23] (see fig. 33). The accuracy of these methods is low in our conditions, about $30 \%$. On average the sound speed was found to be $\langle C\rangle=3.0 \pm 1.0 \mathrm{~cm} / \mathrm{s}$. The dust sound speed $C$ of a dense complex plasma can be estimated by using the relationship

$$
C=\sqrt{\frac{Z T_{i}}{m}}
$$

where $T_{i}$ is the ion temperature, $m$ is the microparticle mass, and $Z$ is the microparticle charge number. For our set of parameters listed above $Z=4000, m=3.2 \cdot 10^{-11} \mathrm{~g}$, assuming the standard values $T_{i}=T_{\text {gas }}=T_{\text {room }} \simeq$ $0.03 \mathrm{eV}$, it yields $C \approx 2.4 \mathrm{~cm} / \mathrm{s}$ in a fairly good agreement with our observations.

As in rf complex plasmas 21] the instability is most probably triggered by the plasma ions $[18,43,51,52$. It is worth noting that, albeit the pressure of $100 \mathrm{~Pa}$ seems very high for waves to occur, the waves observed in our experiments are good 'in-line' with those excited without gas flow 43 , hence indicating a similarity of the excitation mechanism 9 It is straightforward to find that for our set

${ }^{8}$ All pixel intensities of the first image were treated as a scaled red color, of the second one - as green, and, finally, of the third - as blue.

9 The instability is often originated as either the ion-dust streaming instability 43 [51] or the dust-acoustic instability [18,52. 
of parameters the theory predicts the following conditions for the existence of this instability:

$$
\lambda f \simeq C, \quad E \gtrsim E_{c r}=\frac{\gamma_{E p s}}{C} \frac{T_{i}}{|e|} .
$$

Both relationships are of a rather simple physical meaning. The first one merely means that the excitations are of an acoustic type, that agrees fairly well with our findings (fig. 31). The measured frequency of the waveridges $\langle f\rangle=29 \pm 2 \mathrm{~Hz}$ as well as the wavelength $\langle\lambda\rangle=1.1 \pm 0.3 \mathrm{~mm}$ also correspond well to those expected. The second equation introduces an instability threshold $E_{c r}$ which is proportional to the dust damping rate, i.e. it depends on the gas pressure. It is also as intuitively expected. Giving our set of the measured parameters, we obtained $E_{c r} \approx 2.3 \mathrm{~V} / \mathrm{cm}$, that is easy reachable inside the nozzle, also in a fairly good agreement with our observations.

Convection cell. - No wave activity, at least enough intense to be traced, was recorded inside the tail part of the cluster (fig. 2). This most rarefied part of the cloud is dominated by the weak particle drift directed upwards along the gas flow lines close to the nozzle axis 10 and downwards at the periphery of the cluster (see fig. 目 demonstrating the momentary velocity vector field of the drifting particles). In fig. (4it is clear that the particles are forming vortices at the edges of the cluster, developing a single complete circulation cell. By analogy with [36, 39. convection, at least partly, might be caused by a creeping gas flow. The circulation cell size somewhat varies with the gas flow rate. There also might be some variation with height. However, it cannot to be studied in detail in this experiment setup because the parts in the very periphery are poorly accessible via the applied laser illumination. A few traced particles moving back down along the outside of the cloud are shown in fig. 4 .

Convection of the particles, a clearly recognizable feature of their motion close to the nozzle axis, can affect the low frequency oscillations but cannot be the reason for the strong wave excitations, merely because its maximum frequency is much lower than that of the wave-ridges. The maximum angular velocity of conveying particles in the head of the cluster is $\omega_{c} \approx 20-30 \mathrm{~s}^{-1}$, that is 6-7 times lower than that measured for the waves.

Discussion. - In the present work, using a nozzleshaped glass insert in the glass tube plasma chamber of the PK-4 experiment and a gas flow for suspending the particles against gravity, a complicated structure within the microparticle cloud located above the nozzle has been observed and analyzed. The main results of these investigations are: (i) There is an extended stable particle cloud confined above the nozzle showing convection as well as an autowave pattern; (ii) This complicated and stable structure is the result of the various internal and external forces

\footnotetext{
${ }^{10}$ The particle tracks are a bit perturbed by the wave activity located upstream; on average, $V_{\text {drift }}=(4 \pm 1) \mathrm{mm} / \mathrm{s}$.
}

acting on the microparticles, i.e. gravity, longitudinal and radial electric fields in the presence of the nozzle, an applied gas flow from below, the Coulomb interaction between the microparticles, and the interaction of the microparticles with the ions; (iii) The global structure of the main cloud was explained quantitatively by employing the balance of the various forces acting on the particles; (iv) A possible explanation for the convection observed within the main cloud could be creeping gas flow along the glass walls of the chamber and nozzle as also seen in previous investigations with PK-4; (v) The autowaves or self-excited waves observed in the main cloud result from the wellknown ion-dust streaming instability observed in complex plasmas under various conditions.

Conclusion. - To summarize, we have reported a direct observation of a wave pattern disclosed by dense microparticle clusters stably confined in a dc discharge above a de Laval nozzle. The clusters were in an active, marginally unstable state, accompanied by wave excitations. The wave pattern consisted of steep regular wave ridges, fast propagating through the cloud, as well as of low amplitude density fluctuations, originated as dust sound. It is noticeable that varying the cluster position by adjusting the gas flow had no or only little effect on the wave propagation speed. We have argued that the instability is triggered by the plasma ions by means of a well-known ion-dust streaming instability mechanism. The measured space and times scales of the waves are in fairly good agreement with this theory, which supports this claim.

Acknowledgements. - The research leading to these results has received funding from the European Research Council under the European Unions Seventh Framework Programme (FP7/2007- 2013)/ERC Grant Agreement No. 267499, as well as funding by a Marie Curie International Outgoing Fellowship within the 7th European Community Framework Programme, and by DLR (BMBF) under Grants No. 50WP0204, No. 50WM0504, No. 50WM0804, and No. 50WM1150. The authors would like to thank Ch. Rau for his help.

\section{REFERENCES}

[1] G. E. Morfill and A. V. Ivlev, Rev. Mod. Phys. 81, 1353 (2009).

[2] M. Lampe, G. Joyce, G. Ganguli and V. Gavrishchaka, Phys. Plasmas 7, 3851 (2000).

[3] A. P. Nefedov, O. F. Petrov,V. I. Molotkov, V. E. Fortov, JETP Lett. 72, 218 (2000).

[4] M. Kroll, J. Schablinski, D. Block and A. Piel, Phys. Plasmas 17, 013702 (2010).

[5] C.-R. Du, V. Nosenko, S. Zhdanov, H. M. Thomas and G. E. Morfill, EPL 99, 55001 (2012).

[6] M. A. Fink, S. K. Zhdanov, M. H. Thoma,H. Höfner, and G. E. Morfill, Phys. Rev. E 86, 065401(R) (2012).

[7] H. M. Thomas, G. E. Morfill, V. E. Fortov, A. V. Ivlev, V. I. Molotkov, A. M. Lipaev, T. Hagl, H. Rothermel, S. A. 
Khrapak, R. K. Suetterlin, M. Rubin-Zuzic, O. F. Petrov, V. I. Tokarev, and S. K. Krikalev, NJP 10, 033036 (2008).

[8] R. J. Heidemann, L. Couëdel, S. K. Zhdanov, R. K. Sütterlin, M. Schwabe, H. M. Thomas, A. V. Ivlev, T. Hagl, G. E. Morfill, V. E. Fortov, V. I. Molotkov, O. F. Petrov, A. I. Lipaev, V. Tokarev, T. Reiter, and P. Vinogradov, Phys. Plasmas 18, 053701 (2011).

[9] S. K. Zhdanov, M. H. Thoma and G. E. Morfill, NJP 13, 013039 (2011).

[10] P. Ludwig, H. Kählert and M. Bonitz, Plasma Phys. Control. Fusion 54, 045011 (2012).

[11] L. Wörner, C. Räth, V. Nosenko, S. K. Zhdanov, H. M. Thomas, G. E. Morfill, J. Schablinski and D. Block, EPL 100, 35001 (2012).

[12] S. K. Zhdanov, M. H. Thoma, C. A. Knapek, and G. E. Morfill, NJP 14, 023030 (2012).

[13] Lee-Wen Teng, Pei-Shan Tu and Lin I, Phys. Rev. Lett. 90, 24 (2003)

[14] O. Arp, D. Block, A. Piel and A. Melzer, Phys. Rev. Lett. 93, 165004 (2004).

[15] A. Melzer, Plasma Sources Sci. Technol. 10, 303 (2001).

[16] A. Melzer, B. Buttenschön, T. Miksch, M. Passvogel, D. Block, O. Arp and A. Piel, Plasma Phys. Control. Fusion 52, 124028 (2010).

[17] A. Wysocki, C. Räth, A.V. Ivlev, K. R. Sütterlin, H. M. Thomas, S. Khrapak, S. Zhdanov, V. E. Fortov, A. M. Lipaev, V. I. Molotkov, O. F. Petrov, H. Löwen and G. E. Morfill, Phys. Rev. Lett. 105, 045001 (2010).

[18] V. I. Molotkov, A. P. Nefedov, V. M. Torchinski, V. E. Fortov, and A. G. Khrapak, JETP 89, 477 (1999).

[19] M. H. Thoma, H. Höfner, M. Kretschmer, S. Ratynskaia, G. E. Morfill, A. Usachev, A. Zobnin, O. Petrov, V. Fortov, Microgravity Sci. Technol. 18, 47 (2006).

[20] A. Piel, M. Klindworth, O. Arp, A. Melzer, and M. Wolter, Phys. Rev. Lett. 97, 205009 (2006).

[21] M. Schwabe, M. Rubin-Zuzic, S. Zhdanov, H. M. Thomas, and G. E. Morfill, Phys. Rev. Lett. 99, 095002 (2007).

[22] A. Piel, O. Arp, and M. Klindworth, and A. Melzer, Phys. Rev. E 77, 026407 (2008).

[23] M. Schwabe, S. K. Zhdanov, H. M. Thomas, A. V. Ivlev, M. Rubin-Zuzic, G. E. Morfill, V. I. Molotkov, A. M. Lipaev, V. E. Fortov, and T. Reiter, NJP 10, 033037 (2008).

[24] V. Nosenko, S. K. Zhdanov, S.-H. Kim, J. Heinrich, R. L. Merlino and G. E. Morfill, EPL 88, 65001 (2009).

[25] K. O. Menzel, O. Arp, and A. Piel, Phys. Rev. Lett. 104, 235002 (2010).

[26] E. Thomas, Jr., Phys. Plasmas 17, 043701 (2010).

[27] R. Heidemann, S. Zhdanov, K. R. Sütterlin, H. M. Thomas and G. E. Morfill, EPL 96, 15001 (2011).

[28] K. A. Pacha, J. R. Heinrich, S.-H. Kim, and R. L. Merlino, Phys. Plasmas 19, 014501 (2012).

[29] A. Ivlev, H. Löwen, G. E. Morfill, and P. C. Royall, Complex plasmas and colloidal dispersions, in: Series in Soft Condensed Matter 5, Singapore, World Scientific, 320 p. (2012).

[30] L. Couëdel, D. Samsonov, C. Durniak, S. Zhdanov, H. M. Thomas, G. E. Morfill, and C. Arnas, Phys. Rev. Lett. 109, 175001 (2012).

[31] H. B. Bluestein. Tornado Alley: Monster Storms of the Great Plains. NY: Oxford University Press, 180 p. (1999).

[32] G. D. Freier, J. Geophys. Res. 65, 3504 (1960).

[33] J. F. Kok, N. O. Nilton, Geoph. Res. Lett. 33 L19S10
(2006).

[34] J. Carstensen, F. Greiner, L.-J. Hou, H. Maurer, and A. Piel, Phys. Plasmas 16 , 013702 (2009).

[35] H. Kählert, J. Carstensen, M. Bonitz, H. Löwen, F. Greiner and A. Piel, Phys. Rev. Lett. 109, 155003 (2012).

[36] S. Mitic, R. Sütterlin, A. V. Ivlev, H. Höfner, M. H. Thoma, S. Zhdanov and G. E. Morfill, Phys. Rev. Lett. 101, 235001 (2008).

[37] M. Schwabe, L.-J. Hou, S. Zhdanov, A. V. Ivlev, H. M. Thomas and G. E. Morfill, NJP 13, 083034 (2011).

[38] E. Guazzelli. Fluidized beds: from waves to bubbles, p. 24 in: The Physics of Granular Media, Wiley-VCH Verlag Berlin (2004).

[39] M. Schwabe, M. Rubin-Zuzic, S. Zhdanov, A.V. Ivlev, H. M. Thomas, and G. E. Morfill, Phys. Rev. Lett. 102, 255005 (2009).

[40] M. Faraday, Phil. Trans. (London), 121299 (1831).

[41] A. Usachev, A. Zobnin, O. Petrov, V. Fortov, M. Thoma, M. Kretschmer, S. Ratynskaia, R. Quinn, H. Höfner and G. E. Morfill, Czech. J. Phys. 54, C639 (2004).

[42] V. Fortov, G. Morfill, O. Petrov, M. Thoma, A. Usachev, H. Höfner, A. Zobnin, M. Kretschmer, S. Ratynskaia, M. A. Fink, K. Tarantik, Yu. Gerasimov and V. Esenkov, Comments Plasma Phys. Contr. Fusion 47, B537 (2005).

[43] S. A. Khrapak, S. V. Ratynskaia, A. V. Zobnin, A. D. Usachev, V. V. Yaroshenko, M. H. Thoma, M. Kretschmer, H. Höfner, G. E. Morfill, O. F. Petrov and V. E. Fortov, Phys. Rev. E 72, 016406 (2005).

[44] G. E. Morfill, U. Konopka, M. Kretschmer, M. RubinZuzic, H. M. Thomas, S. K. Zhdanov and V. Tsytovich, NJP 8, 7 (2006).

[45] A. Becker, R. Sandheinrich, T. Bretl, IEEE-RSJ Int. Conf on Intel. Robots and Systems, 781 (2009).

[46] P. D. Cha, J. J. Rosenberg, C. L. Dym. Fundamentals of Modeling and Analyzing Engineering Systems, Cambridge, 466p. (2000); R. A. Schilling, S. L. Harris. Fundamentals of Digital Signal Processing Using MATLAB, Toronto: Thompson, 766 p. (2005).

[47] P. S. Epstein, Phys. Rev. 23, 710 (1924).

[48] V. Godyak, R. Lagushenko and J. Maya, Phys. Rev. A 38 (1988).

[49] L. Sirghi, K. Ohe and G. Popa, J. Phys. D: Appl. Phys. 30, 2431 (1997).

[50] S. K. Zhdanov, M. Schwabe, R. Heidemann, R. Sütterlin, H. M. Thomas, M. Rubin-Zuzic, H. Rothermel, T. Hagl, A. V. Ivlev, G. E. Morfill, V. I. Molotkov, A. M. Lipaev, O. F. Petrov, V. E. Fortov, and T. Reiter, NJP 12, 043006 (2010).

[51] R. Bharuthram, H. Saleen, and P. K. Shukla, Phys. Scr. 45512 (1992).

[52] M. Rosenberg, Planet. Space Sci. 41229 (1993). 